\title{
L'AMÉNAGEMENT PISCICOLE DES LACS SUBALPINS ${ }^{(1)}$
}

\author{
par P. VIVIER, \\ Directeur de la Station Centrale d'Hydrobiologie appliquée
}

Il peut paraître téméraire ou illusoire de donner quelques aperçus sur l'aménagement piscicole des lacs subalpins : les données sont sans doute encore trop fragmentaires pour effectuer une synthèse sans critique. Pourtant ces lacs présentent un certain nombre de caractères limnologiques communs d'où il résulte un peuplement piscicole assez semblable, sinon en nombre d'individus et d'espèces, du moins en famille. On peut donc en retirer certaines indications provisoires intéressantes quant à l'aménagement de ces lacs.

\section{I. - CARACTERES LIMNOLOGIQUES.}

Ils jalonnent, on le sait, la chaîne axiale des Alpes, en Autriche, en Allemagne Fédérale, en Suisse, en France, en Italie et en Yougoslavie (Slovenie) ; leur altitude est moyenne, comprise entre 200 et $700 \mathrm{~m}$; ils présentent une grande profondeur, en général supérieure à $100 \mathrm{~m}$. Ce sont, pour l'immense majorité d'entre eux, des lacs oligotrophes, c'est-àdire pauvres en nourriture planctonique, toujours riches en oxygène, même dans les grandes profondeurs, transparents, aux eaux bleues, bleues-vertes ou vertes variant suivant les lacs et les saisons, du no III au $n^{\circ} \mathrm{X}$ de la gamme de Forel-Ule. Ils sont relativement pauvres en phosphore et en azote. Le limon du fond peu fourni en organismes vivants est caractérisé par l'association de la larve du Chironomide Tanytarsus, tandis que le plancton est dépourvu de la larve transparente du Diptère Chaoborus. La beine étant étroite, les végéraux aquatiques supérieurs sont peu abondants sur les bords. Sans doute existe-t-il toutes les gammes d'oligotrophies dans ces lacs et certains d'entre eux les moins profonds, tels que le Lac de Morat $(46 \mathrm{~m})$, sont-ils franchement eutrophes, mais c'est là une exception. Sur les 43 lacs étudiés ici, 10 ont plus de $200 \mathrm{~m}$, 13 de 100 à 200 m., 13 de 50 à 100 m., 7 seulement ont de 30 à $50 \mathrm{~m}$.

(1) Rapport présenté à la $7^{e}$ session du Conseil général des pêches pour la Méditerranée (F. A. O.), Comité des eaux intérieures, Madrid, mars 1963. 
En raison de la profondeur, l'épilimnion se trouve l'été des plus réduits par rapport à l'hypolimnion dont la température reste pratiquement invariable et toujours froide. Pour des lacs de plus de $100 \mathrm{~m}$ de fond, à partir de $30 \mathrm{~m}$ la température ne varie guère dans le cours de l'année $\left(1^{\circ} \mathrm{C}\right.$ dans le Lac du Bourget, $3^{\circ} \mathrm{C}$ dans le Léman). Ainsi cet hypolimnion constitue-t-il un milieu de choix pour les Salmonidés, même de fond (Omble Chevalier, certains Corégones), qui y trouvent en toutes saisons des quantités d'oxygène suffisantes. Il n'en est pas de même pour les Cyprinidés qui ont besoin pour frayer d'eaux relativement chaudes; ceux-ci sont pratiquement cantonnés dans l'épilimnion dont la température varie dans le cours de l'année. Le lac se sépare en deux zones, l'une supérieure à Salmonidés, l'autre inférieure, beaucoup plus importante, domaine exclusif des Salmonidés et de la Lotte, lorsqu'elle existe. On peut comparer un lac subalpin à un étang profond à Cyprinidés, superposé à un lac toujours froid, d'une température inférieure à une dizaine de degrés en toutes saisons.

En outre, ces lacs sont-ils tous, à une ou deux exceptions près (Wörthersee), holomictiques, c'est-à-dire que leurs eaux sont entièrement mélangées au moment du refroidissement hivernal, ce qui est important pour la vie des Salmonidés de fond.

\section{II. - PEUPlement PISGicole.}

Tous ces lacs contiennent un peuplement mixte de :

- Salmonidés,

- Cyprinidés,

- Percidés;

avec, accessoirement, des Gadidés (Lotte) et des Esocidés (Brochet).

Certains de ces lacs peuvent avoir, en outre, des représentants d'autres familles par suite de leur situation particulière. C'est ainsi que le Lac du Bourget contient un Blennidé (Blennius fluviatilis), d'origine méridionale, que des Aloses sédentaires (Paralosa lacustris) occupent une place importante dans les lacs de l'Italie du Nord et qu'on peut trouver des Silures, en petite quantité, ou des Anguilles dans les lacs subalpins d'Autriche, de Suisse et d'Allemagne (Ossiacher See, Lac de Bienne, etc...). L'Anguille n'existe pas dans les lacs français, à l'exception du Lac du Bourget, les communications avec le Rhône étant trop difficiles.

Les Salmonidés sont des poissons de choix, par la délicatesse de leur chair et leur prix élevé ; aussi doivent-il être l'objet d'une attention particulière :

La Truite de lac: Salmo trutta lacustris.

L'Omble Chevalier : Salvelinus alpinus.

Les Corégones, aux nombreuses espèces ou variétés : Coregonus $s p$.

L'Omble Chevalier est représenté par sa variété profondus ; c'est dans ces lacs une espèce essentiellement de fond. Cependant elle n'existe pas partout où elle pourrait croître; en effet, il s'agit-là d'une espèce 
nordique qui a été arrêtée dans sa migration vers le Sud par la chaîne alpine; aussi n'existe-t-elle pas naturellement dans les lacs pourtant très profonds de l'Italie du Nord. Si elle a été importée dans certains d'entre eux (Lac Majeur, Lac de Lugano), on ne la trouve pas dans le Lac de Còme ni dans le Lac de Garde. Il en est de même des Corégones, pour la même raison.

Parmi les Cyprinidés, l'Ablette (Alburnus) et le Gardon (Rutilus) dont leurs différentes espèces sont les plus communes, mais on peut trouver, en moins grande quantité, tous les Cyprinidés (Abramis, par exemple) mais surtout les (yprinidés d'eau courante, tels que le Chevesne (Leuciscus). L'Ablette, poisson essentiellement lacustre, peut être extrêmement abondante (par exemple dans certains lacs italiens, tels que le Lac d'Oseo). Le nombre d'espèces de Cyprinidés est extrêmement variable ( 8 dans le Léman, 13 dans le Lac du Bourget, 11 dans l'Attersee). L'Ablette est plus appréciée des consommateurs que le Gardon. Mais, pour simplifier, nous grouperons toutes ces espèces sous la même rubrique, car si elles sont nombreuses en individus, elles n'ont jamais une grande valeur.

Les Percidés sont toujours représentés, à quelques très rares exceptions près qui mériteraient d'être confirmées (Wörthersee, Zellersee). Quelquefois la Perche, en Europe centrale, est associée au Sandre (Sander lucioperca) qui peut même, comme dans l'Ammersee, la supplanter. Ce sont d'excellents poissons.

La Lotte est très communément répandue ainsi que le Brochet, mais la Lotte est dans les lacs subalpins un poisson de fond, le Brochet restant cantonnè dans les faibles profondeurs, sur les berges abritées. La valeur de ces deux espèces est élevée mais elles ne sont jamais très abondantes.

Mais, si le peuplement mixte ainsi défini se retrouve dans tous ces lacs, à quelques exceptions près, l'importance de ces différentes catégories de poissons y est-elle fort différente. La profondeur, la facilité des communications avec les émissaires, la pureté de l'eau, les modes d'exploitation de la pêche sont autant de facteurs qui modifient la proportion relative de ces catégories.

Dans tels de ces lacs, d'une oligotrophic prononcée, c'est l'Omble Chevalier qui sera l'espèce caractéristique (Lac de Zoug, par exemple); à l'autre bout de la chaîne, dans les lacs eutrophes, comme ceux de Morat ou d'Halvill, ce sera, au contraire, la Perche et les Cyprinidés. Mais dans la grande majorité des cas, les Corégones et les Percidés constitueront les espèces dominantes. La proportion relative de ces deux groupes peut varier dans le temps et nous verrons comment il est possible de la modifier par des aménagements appropriés.

\section{PROPORTIONS RELATIVES}

\section{DES DIFFERENTS GROUPES DE POISSONS.}

Las grands lacs subalpins étant en général l'objet d'une pêche professionnelle contrôlée, on possède des statistiques des services compétents, des renseignements sur les espèces de poissons capturés annuelle- 
ment. Bien entendu, ces statistiques ne sauraient donner une indication absolue de l'importance de ces espèces, certaines d'entre elles pouvant ètre négligées par la pèche. Elles donnent néanmoins de précieuses indications sur l'importance relative des espèces et mème sur le rendement réel du lac, lorsque le pêcheur professionnel est assuré qu'il ne sera pas fait usage de ces statistiques dans un but fiscal. Les captures de la pêche sportive échappent à ces statistiques, mais elles sont peu appréciables, à côté des prises de la pêche professionnelle et peuvent être négligées. Dans les eaux suisses du Lac de Constance, par exemple, les statistiques de la pêche sportive donnaient, en $1959: 32.225 \mathrm{~kg}$ pour $352.689 \mathrm{~kg}$, soit un peu moins du dixième.

Dans les statistiques que nous allons étudier et qui concernent les lacs d'une certaine importance, il ne sera donc fait état que de la pêche professionnelle, la plus facile à contrôler.

\section{Lacs à Corégones et à Perches.}

Ces statistiques sont particulièrement bien tenues dans les lacs suisses et dans le Léman.

Voici, par exemple, pour 1960, celles du Lac de Neuchatel (1).

\begin{tabular}{|c|c|c|c|}
\hline Truite & 9.070 & $\mathrm{~kg}$ & $2,04 \%$ \\
\hline Omble Chevalier .... & 58 & - & $0,01-$ \\
\hline Corégone $\ldots \ldots \ldots$ & 257.340 & - & $58,14-$ \\
\hline Perche & 107.138 & - & $24,20-$ \\
\hline Lotte & 1.378 & -- & $0,31-$ \\
\hline Brochet & 4.303 & - & $0,97-$ \\
\hline Cyprinidés & 63.314 & - & $14,30-$ \\
\hline Total & 442.601 & $\mathrm{~kg}$ & $99,97 \%$ \\
\hline
\end{tabular}

Et voici, pour 1961, celles concernant les eaux françaises du Léman (2).

\begin{tabular}{|c|c|c|c|c|c|}
\hline & Truite & 8.961 & $\mathrm{~kg}$ & $1,60 \%$ & \\
\hline & Omble ('hevalier & 17.011 & - & $3,03-$ & $31,73 \%$ \\
\hline & Corégone ..... & 151.745 & - & $27,10-$ & \\
\hline & Perche . & 243.461 & - & 43,48 & \\
\hline & Lotte ... & 15.309 & - & $2,73-$ & \\
\hline & Brochet ....... & 4.939 & - & $0,88-$ & \\
\hline & Ablette .......... & 39.580 & - & 7,07 & \\
\hline des & Gardon et autres ... & 78.871 & - & $14,10-$ & $21,17 \%$ \\
\hline & Total & 559.8 & $\mathrm{~kg}$ & $99,99 \%$ & \\
\hline
\end{tabular}

(1) Quartien (A.), "Le rendement de la pêche dans le Lac de Neuchatel en 1960 ", Le Pêcheur et le Chasseur suisse, $n^{\circ} 2,1961$, p. 53.

(2) Statistiques de l'Inspection des Eaux et Forêts de 'Thonon (non imprimées). 
Et, en 1959, dans les eaux suisses dı Lac de Constance (Bodensee), sur $352.689 \mathrm{~kg}$ pêchés, les Corégones intervenaient pour $57 \%$, suivis ensuite par la Perche et les Cyprinidés.

Si la Perche domine les Corégones dans le Léman, ces trois groupes constituent à eux seuls $90 \%$ des captures annuelles de ces trois lacs.

Les Corégons occupent une place importante dans les lacs subalpins suisses puisqu'ils représentent $41 \%$ en quantité et $46 \%$ en valeur des poissons pêchés dans le pays. Dans le I ac de Thoune par exemple, sur $42.443 \mathrm{~kg}$ de poissons pêchés, $93,9 \%$ sont des Corégones (1). Dans les Lacs de Brienz, de Wallenstadt et des Quatre-Cantons, il y a encore, d'après A. Mathey Doret (2), 80 ou $90 \%$ de Corégones; s'ils n'ont pu être maintenus que grâce à des déversements d'alevins dans les Lacs de Zurich, de Zoug et de Sempach, ils occupent encore dans le premier de ces lacs une place importante puisque sur $140 \mathrm{t}$ de poissons pèchés en 1949, il y avait $65 \mathrm{t}$ de Corégones, soit $47 \%$.

\section{Lacs à Aloses et à Cyprinidés.}

Les lacs italiens, protégés des invasions nordiques par les Alpes, sont dépourvus des Salmonidés originaires du Nord de l'Europe, tels que l'Omble Chevalier et les Corégones. Mais ces espèces ont été importées et se sont acclimatées dans certains d'entre eux. Par contre, ces lacs sont caractérisés par la présence d'une Alose sédentaire, l'Agone (Paralosa lacustris) qui y joue un rôle important.

Voici par exemple la statistique des pêches du Lac Majeur (eaux suisses), en 1954 (3).

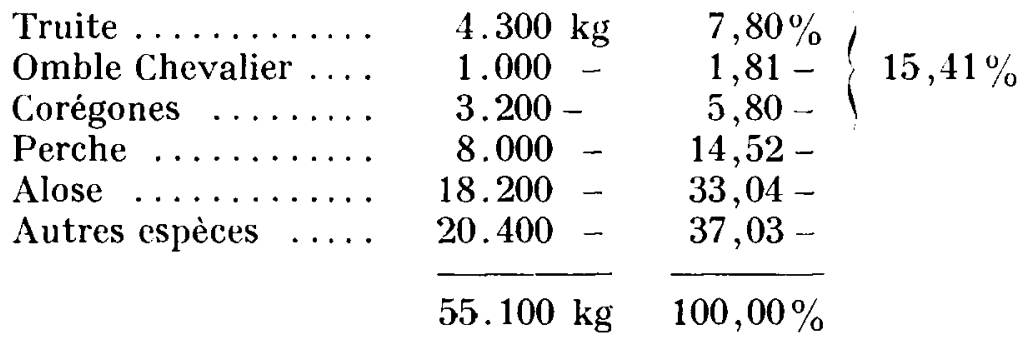

Depuis lors les Corégones seraient en augmentation dans ce lac (4), mais je n'ai pu avoir de statistiques.

L'Alose et les autres espèces (Cyprinidés en majeure partie) constituent $70 \%$ des captures.

(1) Colloque d'Économie piscicole des lacs à Gorégones, compte rendu ronéotypé édité par la Station de Recherches lacustres de Thonon, 1954, 56 pages, "Exposé du Dr Roth", p. 26.

(2) Colloque d'Économie piscicole des lacs à Corégones, p. 25.

(3) Le Pêcheur et le Chasseur suisse, $n^{0} 2$, novembre 1956, p. 390.

(4) Le Pêcheur et le Chasseur suisse, $\mathrm{n}^{0}$ 9, septembre 1959, p. 317. 
Dans les eaux suisses du Lac de Lugano, la même année (1954), les prises étaient constituées ainsi :

\begin{tabular}{lrr|r} 
Truites $\ldots \ldots \ldots \ldots$ & $400 \mathrm{~kg}$ & $0,63 \%$ & \\
Omble Chevalier $\ldots \ldots$ & $500-$ & $0,79-$ & $4,58 \%$ \\
Corégones $\ldots \ldots \ldots$ & $2.000-$ & $3,16-$ & \\
Perche $\ldots \ldots \ldots \ldots$ & $1.200-$ & $6,65-$ & \\
Alose $\ldots \ldots \ldots \ldots$ & $16.000-$ & $23,35-$ \\
Autres espèces $\ldots \ldots$ & $40.000-$ & $65,42-$ \\
\cline { 2 - 2 } & $63.100-$ &
\end{tabular}

Bien que les "autres espèces" ne soient pas désignées dans cette rubrique, les Cyprinidés en forment la majeure partie; avec les Aloses ils constituent $\mathbf{8 8 , 7 7 \%}$ des captures. vants :

Ainsi donc, dans ces lacs, les groupes dominants sont-ils les sui-

$$
\begin{aligned}
& \text { Cyprinidés ; } \\
& \text { Clupéidés (Alose). }
\end{aligned}
$$

Le Lac de Garde fait exception. Dans ce lac les Salmonidés occupent une place beaucoup plus importante (32,8\% du peuplement) (1). En 1955, la situation de la pêche était la suivante, en ce qui concerne cette famille :

$$
\begin{array}{lrr}
\text { Truites lacustres } \ldots . & 4.357 \mathrm{~kg} & 1,42 \% \\
\text { "Carpione } "(2) \ldots \ldots & 24.814 \underset{\mathrm{kg}}{-} & 8,12- \\
\text { Corégones } \ldots . . \ldots & 71.076 \mathrm{~kg} & 23,26-
\end{array}
$$

Ce qui fait, pour une superficie de $370 \mathrm{~km}^{2}$, un rendement de $8 \mathrm{~kg} / \mathrm{ha}$ en salmonidés seulement.

\section{IV. - LE RENDEMENT ANNUEL ET SON AMÉLIORATION.}

Le but d'un aménagement piscicole rationnel consiste dans la détermination du meilleur rendement annuel compatible avec les possibilités biologiques du lac considéré. Kreitrmans (1933) (3), dans une étude fort intéressante, a bien précisé cet objectif : "Il s'agit, en principe, écrit-il, de connaître le peuplement piscicole et son rendement, de déterminer et de discuter les circonstances qui peuvent influer sur la productivité, d'en déduire un peuplement idéal pour le rendement maximum et, enfin, de fixer les moyens par lesquels ce peuplement sera obtenu."

(1) S. Merlot, "La pêche et le repeuplement en Salnonidés dans le Lac de Garde (Conseil général des pêches pour la Méditerranće (F. A. O.) ", Débats el Documents techniques, no 4, 1957, pp. 257-267.

(2) Le "Carpione " est morphologiquement semblable à la Truite lacustre, mais en diffère par la croissance et par l'existence d'une double période annuelle de reproduction (en décembre-janvier et entre le 15 juillet et le 15 août). Certains auteurs le considèrent comme nne variété du Salmo lacustris, d'autres en font une espèce distincte.

(3) Kaeitmann (L.), "De l'aménagement piscicole des lacs ", Revue des Eealux et Forêts, janvier 1933, pp. 4-18. 


\section{$1^{\circ}$ Variation du rendement pondéral annuel.}

Les statistiques permettent d'avoir une idée suffisamment précise sur les rendements annuels des lacs et sur leurs variations. Tel est, par exemple, le cas du Léman qui, lac à Corégones, était devenu lac à Perche vers 1920 à la suite de la multiplication d'un filet nouveau, dit " grand pic »; actuellement, comme on l'a vu plus haut, c'est un lac mixte à Corégones et à Perche. Voici d'ailleurs le rendement annuel du Léman, en 1897, 1922 et 1961 :

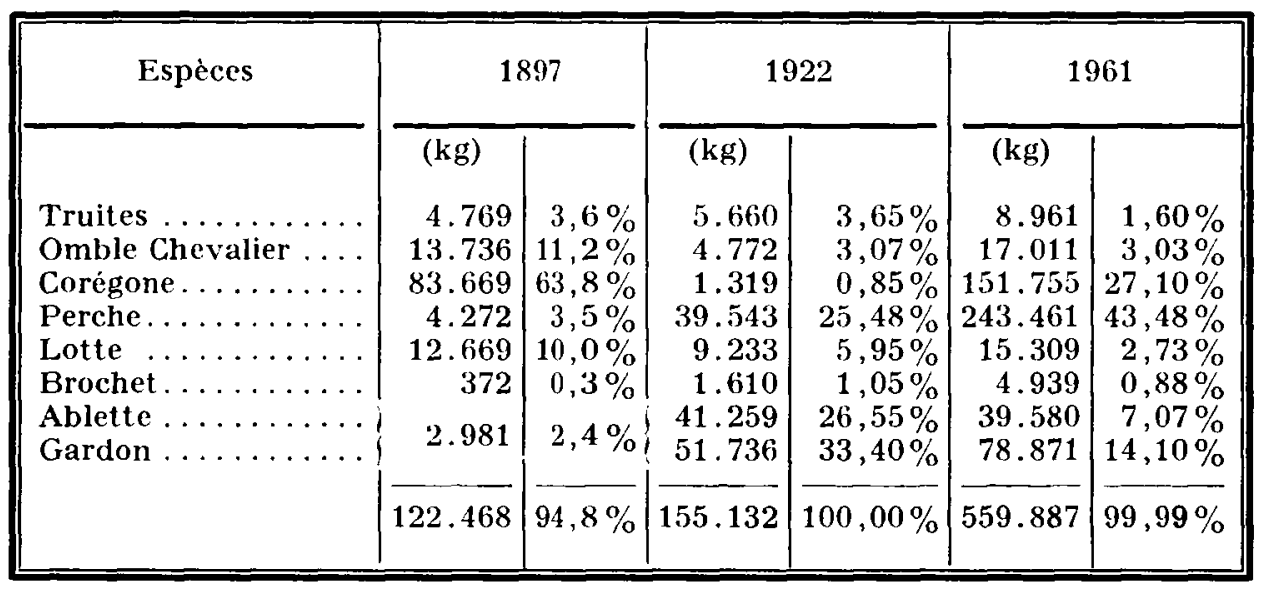

Une première constatation s'impose d'abord : c'est l'augmentation progressive des captures qui se sont multipliées par quatre sans que, semble-t-il, le lac s'appauvrisse. Il faut y voir non seulement les résultats heureux des repeuplements artificiels en Corégones et en Omble Chevalier, mais encore le résultat de l'eutrophisation progressive du Léman (1), favorable au développement des Perches et des Cyprinidés. Les résultats du repeuplement sont surtout manifestes chez les Corégones et le Colloque franco-suisse d'économie piscicole des lacs à Corégones, tenu à Thonon, le 10 mars 1954, l'a confirmé d'une façon générale (2). La capture de géniteurs, nécessairement sacrifiés, au moyen de filets maillants, en période de fermeture par des pêches exceptionnelles, ne présente pas d'inconvénients, étant donné le nombre d'œufs pondus par chaque femelle; de plus elle permet l'élimination de vieux sujets inutiles.

\section{$2^{\circ}$ Le rendement pondéral annuel à l'hectare.}

Lacs à Corégones et à Perches. - Le rendement pondéral annuel pour les grands lacs subalpins oligotrophes à Corégones et Perches est assez semblable; il est facile de le calculer :

(1) Eutrophisation mise en relicf par les travaux de la Commission francosuisse de protection du Léman contre la pollution, 1961, ronéotypé.

(2) Op. cit. 
Dans le Lac Léman (eaux françaises), occupant une superficie de $239 \mathrm{~km}^{2}$, il s'élevait en 1961, pour une production de $559.887 \mathrm{~kg}$, à $23,43 \mathrm{~kg} / \mathrm{ha}$.

Dans le Lac de Neuchatel (en 1960), qui mesure $216 \mathrm{~km}^{2}$, pour une production de $442.601 \mathrm{~kg}$, il était de $20,49 \mathrm{~kg} / \mathrm{ha}$.

Dans le Lac de Constance (eaux suisses), dont la surface est de $176,54 \mathrm{~km}^{2}$ et la production de $352.689 \mathrm{~kg}$, il montait, en 1959 , à $19,98 \mathrm{~kg} / \mathrm{ha}$.

Il est remarquable que, pour ces trois lacs semblables et dont les statistiques sont tenues sérieusement, les résultats concordent parfaitement : le rendement est d'une vingtaine de kilogrammes par hectare et par an.

Il est à noter que ce rendement est en augmentation dans ces trois lacs et qu'il était en 1930 de :

$16,54 \mathrm{~kg} / \mathrm{ha} / \mathrm{an}$ dans le Léman

12,90 - dans le Lac de Neuchatel

8,00 - dans le Lac de Constance (eaux suisses) (1).

Au début du siècle, il était inférieur à $10 \mathrm{~kg}$.

Quelles sont les causes de ce rendement plus élevé ? Est-il dù à des modes de pèche plus perfectionnées ou à une eutrophisation progressive de ces lacs? Il est certain que la teneur en phosphates s'est accrue, qu'ils proviennent de la population en augmentation constante sur les bords, des détergents dont ils servent d'adjuvants ou des cultures. Il semble bien que ce soit cet enrichissement du lac causé par la pollution qui soit la cause principale de cette augmentation. L'eutrophisation est pour l'instant bénéfique pour le pêcheur, mais l'altération qu'elle apporte au lac, par ailleurs, risque d'ètre de la plus grande gravité pour le tourisme et, finalement, plus tard pour la pêche elle-même.

Les modes de pêche se sont perfectionnés depuis un demi-siècle, les filets en nylon sont plus "pêchants " que les autres et le rendement s'est trouvé par là même amélioré. Quant à la pisciculture artificielle, elle ne peut avoir contribué à cette amélioration que pour les espèces nobles. Corégones surtout, mais elle ne saurait expliquer l'accroissement des autres espèces, Perches et Cyprinidés notamment.

Lacs à Aloses et à Cyprinidés. - En se référant aux statistiques citées plus haut, on trouve en 1954 , un rendement de :

$13,15 \mathrm{~kg}$ pour les eaux suisses du Lac Majeur, d'une superficie de $41,90 \mathrm{~km}^{2}$;

$19,85 \mathrm{~kg}$ pour les eaux suisses du Lac de Lugano $\left(31,78 \mathrm{~km}^{2}\right)$.

Le rendement, là encore, est assez voisin des rendements précédemment indiqués.

Lacs à Omble Chevalier. - Le rendement pondéral est faible; d'après Haempel (1930) il serait de $6 \mathrm{~kg} / \mathrm{ha}$ dans le Lac de Zoug, chiffre admis d'autre part par Smolian pour les lacs de ce type.

(1) D'après KreitmanN, op. cit. 
Lacs à Cyprinidés et à Perches. - Les rendements sont fort différents suivant les lacs; ils varient avec l'intensité de la pêche, les Cyprinidés, de peu de valeur alimentaire n'étant appréciés que dans les pays pauvres. C'est ainsi que si l'on en croit l'A venire de la pesca, cité par L. HaEmpel, le Lac d'Iseo aurait produit en $1922: 298.000 \mathrm{~kg}$ pour une superficie de $56,34 \mathrm{~km}^{2}$, soit un rendement à l'hectare de $52 \mathrm{~kg}$ et, en 1923 , le rendement était très supérieur. Il était dù à l'extrême abondance des Ablettes.

Par contre, le lac eutrophe de Morat ne donnait, en 1920 , que $6 \mathrm{~kg} / \mathrm{ha}$, d'après le même auteur (13.958 kg pour $2.282 \mathrm{ha}$ ).

\section{$3^{0}$ Le rendement financier annuel.}

Le rendement financier annuel, le seul en définitive qui importe, est très variable suivant la qualité du poisson pêché. En France, les prix à la production (1962) varient entre $12 \mathrm{~F}$ le kilogramme pour la Truite de lac et l'Omble Chevalier, poissons nobles par excellence et $1 \mathrm{~F}$ le kilogramme pour le Gardon. Il est donc bien évident que partout où cela est possible, le but de l'aménagiste est de développer les poissons nobles, c'est-à-dire 'Truite et Omble Chevalier, au détriment des poissons vulgaires, même si, comme cela est de règle, les rendements pondéraux doivent être diminués.

Lorsqu'il s'agit, ainsi qu'il arrive fréquemment, de lacs mixtes à Corégones et à Perches, la solution est moins évidente $a$ priori. Corégones et Perche sont appréciés les uns comme les autres et vivent en compétition. Mis à part les habitudes locales, les débouchés économiques et les goûts des consommateurs, un petit calcul va cependant nous montrer que, dans ces lacs, les plus nombreux, il faut accroître les Corégones au détriment de la Perche à tendance envahissante.

\begin{tabular}{|c|c|c|c|c|c|}
\hline \multirow{2}{*}{ Espèces } & \multirow{2}{*}{$\begin{array}{l}\text { Prix } \\
\text { actuels }\end{array}$} & \multicolumn{2}{|c|}{1922} & \multicolumn{2}{|c|}{1961} \\
\hline & & $\begin{array}{l}\text { Proportion } \\
\text { des espèces }\end{array}$ & Valeur & $\begin{array}{l}\text { Proportion } \\
\text { des espèces }\end{array}$ & Valeur \\
\hline & (F) & $(\mathrm{kg})$ & (F) & $\overline{(\mathrm{kg})}$ & $(F)$ \\
\hline Truite et Omble .. & 12 & 0,672 & 8,064 & 0,463 & 5,556 \\
\hline Corégones...$\ldots$. & 4 & 0,085 & 0,340 & 2,710 & 10,840 \\
\hline Perche ... & 2 & 2,548 & 5,096 & 4,348 & 8,696 \\
\hline Lotte $\ldots \ldots \ldots \ldots$ & 5 & 0,595 & 2,975 & 0,273 & 1,365 \\
\hline Brochet .. & 7 & 0,105 & 0,735 & 0,089 & 0,623 \\
\hline Ablette $\ldots \ldots \ldots \ldots$ & 1,5 & 2,655 & 3,982 & 0,707 & 1,060 \\
\hline \multirow[t]{2}{*}{ Gardon .......... } & 1 & 3,340 & 3,340 & 1,410 & 1,410 \\
\hline & & 10,000 & 24,532 & 10,000 & 29,550 \\
\hline
\end{tabular}


En nous basant sur les statistiques de 1922 et de 1961 , il est aisé, pour le Léman, de trouver le rapport financier de $10 \mathrm{~kg}$ de poissons pêchés, d'une part, en 1922, lorsque les Corégones avaient pour ainsi dire disparu, d'autre part en 1961 où coexistent Corégones et Perches. Pour permettre des comparaisons, on affectera les prix actuels aux captures de 1922.

On voit que, pour $10 \mathrm{~kg}$ de poissons pêchés, le prix moyen a passé de $24,532 \mathrm{~F}$ à $29,550 \mathrm{~F}$, soit environ une augmentation de $4 \mathrm{~F}$, les Corégones et les Perches formant en 1961 les $2 / 3$ de ce prix. Il faut noter cependant que cette augmentation serait beaucoup plus sensible si la proportion des Perches/Corégones était inversée et si, au lieu de $4,348 \mathrm{~kg}$ de Perches, on pêchait le même poids de Corégones. Au lieu de 19,536 F, le prix pour ces deux groupes passerait à $22,812 \mathrm{~F}$ et le prix total des $10 \mathrm{~kg}$ à 32,826 (au lieu de 29,550). C'est donc vers cette augmentation des Corégones que doivent se poursuivre les efforts de l'administration.

$\mathrm{Si}$, au lieu de considérer le prix de $10 \mathrm{~kg}$ de poissons pèchés, on examine le tonnage global qui s'est accru, l'augmentation financière est plus sensible encore. Les captures ayant passé de $155.197 \mathrm{~kg}$ en 1922 , à $559.887 \mathrm{~kg}$ en 1961 , le produit financier de la pêche est monté de $380.569,82 \mathrm{~F}$ à $1.642 .466,08 \mathrm{~F}$, soit plus de 4 fois.

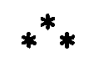

Cet exemple, emprunté au Lac Léman, montre qu'il est d'un intérêt hautement économique d'aménager scrupuleusement nos lacs subalpins en vue du meilleur profit. Celui-ci n'est pas déterminé au hasard. Il peut être modifié par la réglementation, d'une part et par la pisciculture de l'autre. La tenue scrupuleuse des statistiques de pêche permet de contrôler les résultats. Encore faut-il que les États intéressés attachent, comme cela se pratique surtout en Suisse, de l'intérêt à l'aménagement rationnel de ces lacs subalpins si intéressants pour la pêche. 
$-146-$

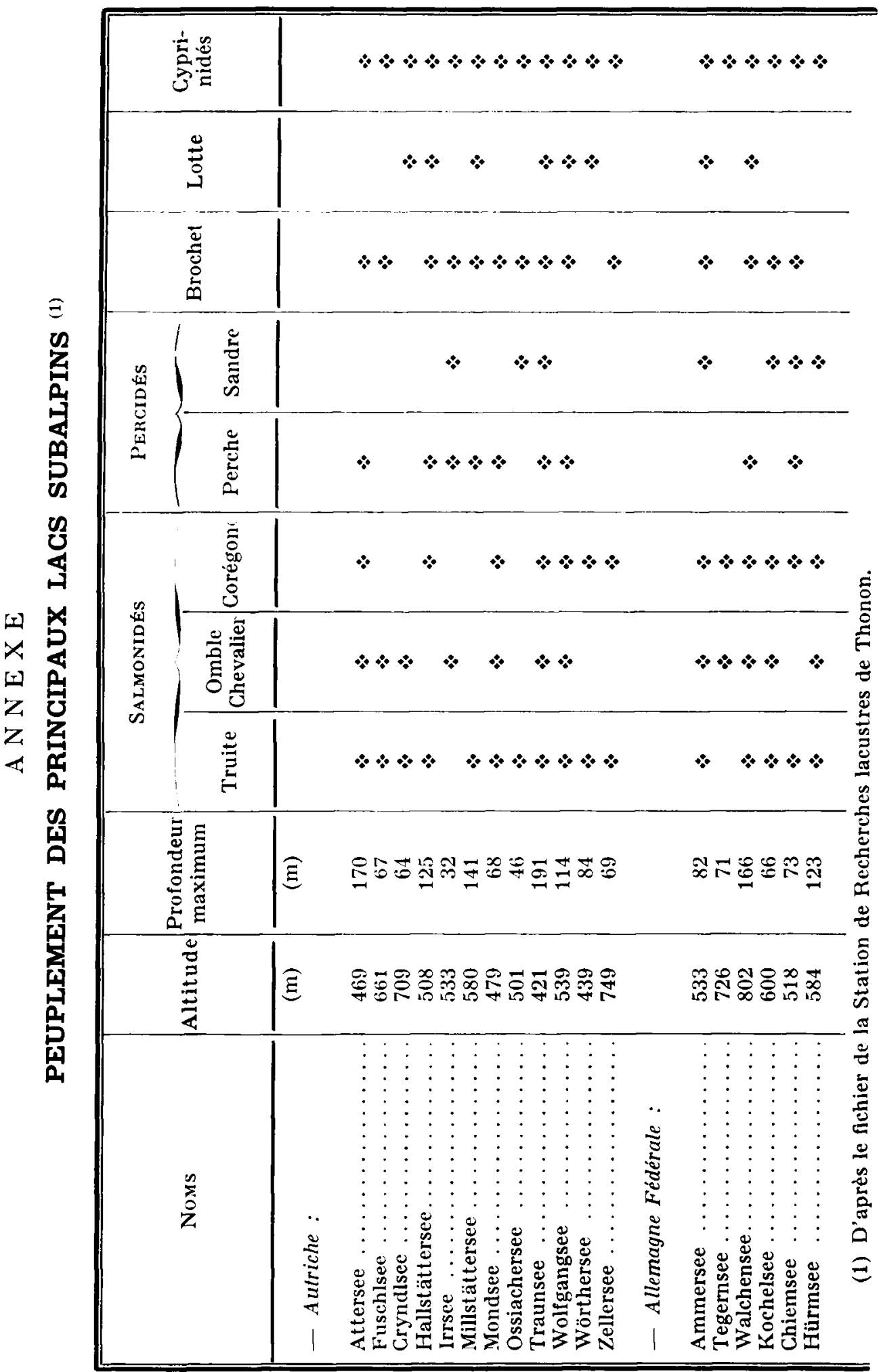


$-147-$

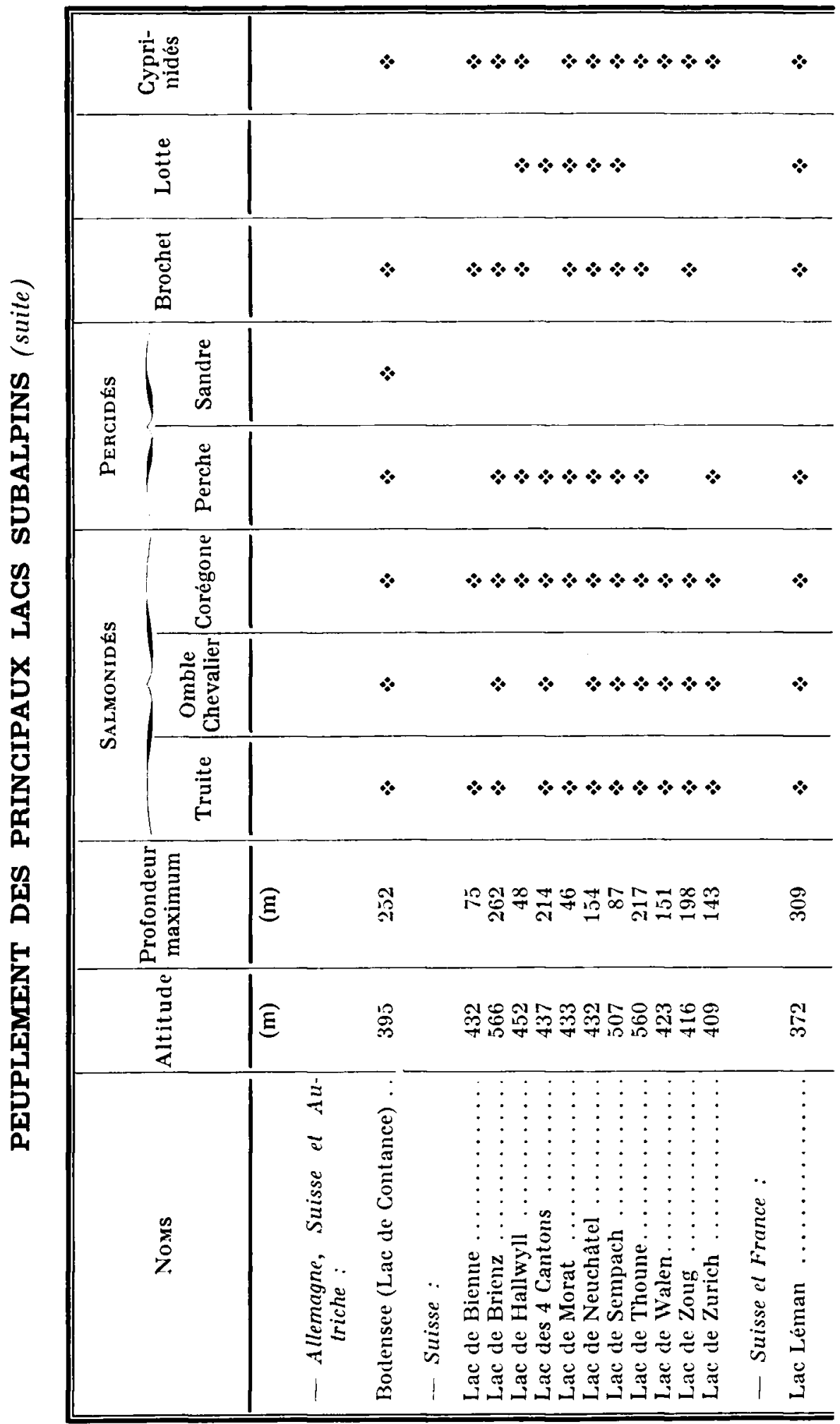


$-148-$

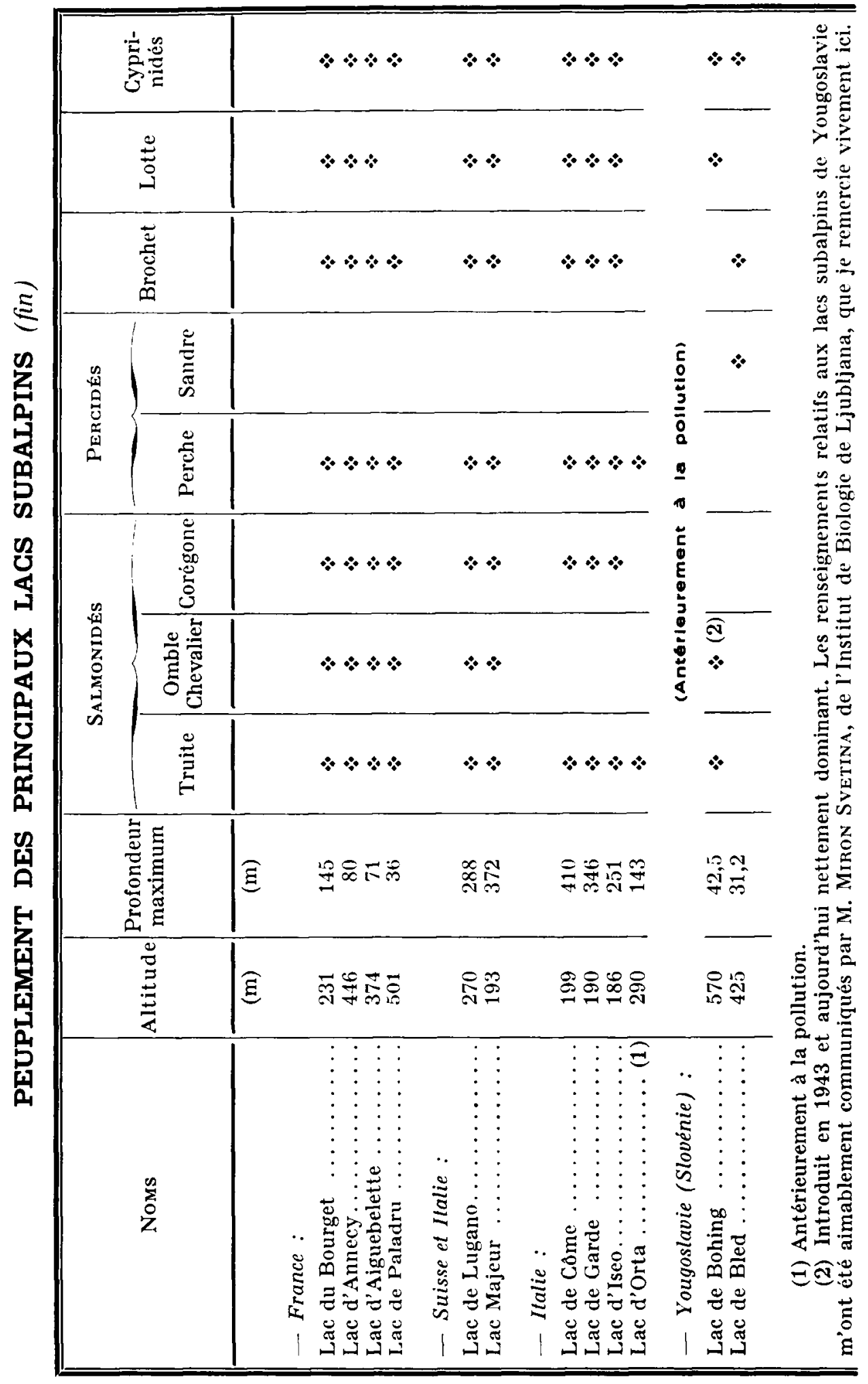




\section{Résumé}

Les lacs subalpins sont d'altitude moyenne (de 200 à $700 \mathrm{~m}$ ), généralement profonds (plus de $100 \mathrm{~m}$ ) et oligotrophes. Ils présentent l'été un épilimnion chaud se superposant à un hypolimnion toujours froid.

Aussi abritent-ils aussi bien des Salmonidés que des Cyprinidés et des Percidés, avec accessoirement des Gadidés (Lotte) et des Esocidés (Brochet). Les lacs de l'Europe centrale contiennent en outre des Siluridés (Silurus glanis) et ceux d'Italie du Nord (à l'exception du Lac de Garde) des Clupéidés (Paralosa lacustris).

Les lacs les plus oligotrophes sont les lacs à Omble-Chevalier (Salvelinus alpinus), les plus eutrophes des lacs à Cyprinidés et à Perche (Lac de Morat). Le plus grand nombre de ces lacs sont cependant des lacs à Corégones et à Perche (Léman, Neuchâtel, Constance, etc...).

Le rendement pondéral annuel varie avec le mode d'exploitation. Par exemple, de 1897 à 1961, dans les eaux françaises du Lac Léman, les captures annuelles en poids se sont accrues de 4 fois; mais alors que les Corégones représentaient $63,8 \%$ de ces captures en 1897 , ils n'atteignaient pas $1 \%$ en 1922, pour remonter à $27,1 \%$ en 1961 .

Ce rendement est, à l'hectare, d'une vingtaine de kilogrammes pour les lacs à Corégones et à Perche, plus faible pour les lacs à Omble-Chevalier et plus fort pour les lacs à Cyprinidés et à Perche.

Le rendement financier annuel varie non seulement avec l'abondance des captures mais aussi avec la qualité du poisson pêché. Aussi dans le Lac Léman le prix moyen de $10 \mathrm{~kg}$ de poisson pếchés a augmenté de $4 \mathrm{~F}$, de 1922 à 1961, grâce à une plus grande abondance relative des Corégones. 